\title{
Development of multi-dimensional body image scale for Malaysian female adolescents.
}

\begin{abstract}
The present study was conducted to develop a Multi dimensional Body Image Scale for Malaysian female adolescents. Data were collected among 328 female adolescents from a secondary school in Kuantan district, state of Pahang, Malaysia by using a self-administered questionnaire and anthropometric measurements. The self-administered questionnaire comprised multiple measures of body image, Eating Attitude Test (EAT-26; Garner \& Garfinkel, 1979) and Rosenberg Self-esteem Inventory (Rosenberg, 1965). The 152 items from selected multiple measures of body image were examined through factor analysis and for internal consistency. Correlations between Multi-dimensional Body Image Scale and body mass index (BMI), risk of eating disorders and self-esteem were assessed for construct validity. A seven factor model of a 62-item Multi-dimensional Body Image Scale for Malaysian female adolescents with construct validity and good internal consistency was developed. The scale encompasses 1) preoccupation with thinness and dieting behavior, 2) appearance and body satisfaction, 3) body importance, 4) muscle increasing behavior, 5) extreme dieting behavior, 6) appearance importance, and 7) perception of size and shape dimensions. Besides, a multidimensional body image composite score was proposed to screen negative body image risk in female adolescents. The result found body image was correlated with BMI, risk of eating disorders and self-esteem in female adolescents. In short, the present study supports a multi-dimensional concept for body image and provides a new insight into its multi-dimensionality in Malaysian female adolescents with preliminary validity and reliability of the scale. The Multi-dimensional Body Image Scale can be used to identify female adolescents who are potentially at risk of developing body image disturbance through future intervention programs.
\end{abstract}

Item Type: Article

Keyword: Body image; Factor analysis; Body mass index; Eating disorders; Self-esteem. 